\title{
On a property of the stable or conditionally stable solutions of systems of non-linear differential equations.
}

By P. Turàn (in Budapest)

\author{
A Giovanni Sansone nel suo $70^{\circ}$ compleanno.
}

Sammary. - Qualitatively expressed we assert the following. If the equation (1.1)-(1.2) fulfills the restriction (1.6)-(1.7)(1.8) whic are less restrictive than usual, then all solutions with norms tending to $O$ with $\frac{1}{t}$ cannot be a to small $*$ in an arbitrary interval in $t \geqq 0$ with fixed length.

1. As well-known, the stability-problem of a non-linear system can be reduced to the investigation of the stability of $X(t) \equiv 0$ concerning the system

$$
\frac{d X}{d t}=\boldsymbol{A} X+W(X, t)
$$

with

$$
W(0, t) \equiv 0 ;
$$

here stand $X=X(t)$ and $W(X, t)$ for the $n \times 1$ colum-vectors

$$
X(t) \equiv\left(\begin{array}{c}
x_{1}(t) \\
x_{2}(t) \\
\vdots \\
x_{n}(t)
\end{array}\right), \quad W(Y, t)=\left(\begin{array}{c}
w_{1}\left(y_{1}, \ldots, y_{n}, t\right) \\
w_{2}\left(y_{1}, \ldots, y_{n}, t\right) \\
\vdots \\
w_{n}\left(y_{1}, \ldots, y_{n}, t\right)
\end{array}\right)
$$

and $\boldsymbol{A}$ stands for an $n \times n$ matrix with complex elements

$$
A=\left(\begin{array}{cc}
a_{11} \ldots & a_{1 n} \\
\vdots & \vdots \\
a_{n 1} \ldots & a_{n n}
\end{array}\right)=\left(a_{i n}\right) .
$$

It was discovered for certain systems by LIAPounofF and PoInCané and for more general systems extended by Comion and Perron ( ${ }^{1}$ ) that e.g. if all solutions $X_{1}(t)$ with sufficiently small $\left\|X_{1}(0)\right\|$ of the system (1.1) - (1.2) tend to 0 for $t \rightarrow+\infty$ then they cannot be «too near» to 0 for $t>t_{0}$,

(1) O. Perron, Uber Stabilität und asymptotisches Verhalten der Integrale von Differentialgleichungssysteme, "Math. Zeitschrift \&, Bd. 29, p. 129-160. 
whatever large we choose $t_{0}$. More exactly they found that in this case for all solutions $X(t)$ of the system $(1.1)-(1.2)$ the relation

$$
\varlimsup_{t \rightarrow+\infty} \frac{\log \|X(t)\|}{t}=\operatorname{Re} \lambda_{v}
$$

holds with a $\vee$ where $\lambda_{y}$ stand for the characteristic roots of $A$ and

$$
\|X(t)\|=\left(\sum_{\nu=1}^{n}\left|x_{\nu}(t)\right|^{2}\right)^{1 / 2}
$$

Hence in this case for each such solutions and an arbitrary small $\varepsilon>0$ there is a sequence

such that

$$
t_{1}<t_{2}<\ldots \rightarrow+\infty
$$

$$
\left\|X\left(t_{v}^{*}\right)\right\|>e^{\left(\operatorname{Re} \lambda_{n}-\varepsilon\right) t_{v}} \quad v=1,2, \ldots
$$

where

$$
(0>) \operatorname{Re} \lambda_{1} \geqq \operatorname{Re} \lambda_{2} \geqq \cdots \geqq \operatorname{Re} \lambda_{n} .
$$

These results however give no information about the distribution of these $t_{v}$-values so that the picture it furnishes about the «Endverlauf» of solutions «near» to a stable solution remains rather incomplete. Even the possibility cannot be excluded that for a solution $X_{1}(\dot{t}) \neq 0$ of the system (1.1)-(1.2) for arbitrary large segments $X_{1}(t)$ vanishes. A. more refined theory would be beside the possible technical applications the more desirable since a systematic negative use of it would mean a standard device in the treatment of such problems that a certain system

$$
x_{1}(t), x_{2}(t), \ldots, x_{n}(t)
$$

of functions cannot satisfy certain types of systems of differential equations. For the treatment of these questions I shall return elsewhere.

In this note I shall show that essentially the inequality (1.3) is satisfied in every interval of a fixed length of the positive $t$-axis and this holds for a more general class of systems (1.1)-(1.2). More exactly we consider systems (1.1)-(1.2) for which - denoting the characteristic roots of $A$ by $\lambda_{1}, \lambda_{2}, \ldots$ $\ldots, \lambda_{n}$ - we have

$$
\operatorname{Re} \lambda_{n}=\min _{j} \operatorname{Re} \lambda_{j}=L<0 .
$$

Let $\Delta$ be so large that $\Delta>1$ and

$$
5 n(2 e(\Delta+1))^{2 n} e^{2 L \Delta} \leqq 1
$$


and the $W(Z, t)$ in the equations (1.1) such that in a halfeylinder of the $(Z, t)$-space

$$
\|Z\| \leqq c_{1}, \quad t \geqq 0 \quad\left(c_{1} \text { positive constant }\right)
$$

the inequality

$$
\|W(Z, t)\| \leqq \frac{e^{-2 n\|A\|\left(\Delta^{2}+\Delta\right)}}{3 V \bar{n} \sqrt{\nu^{2} \Delta+\Delta}}\|Z\|
$$

holds; here $\|A\|$ stands for $\max _{i, k}\left|a_{i k}\right|$. Then we assert the following

Theorem. - If $Y(t)$ with $\lim _{t \rightarrow+\infty}\|Y(t)\|=0$ is a solution of the system (1.1)-(1.2) satisfying the restrictions (1.6)-(1.8), then we have for all sufficiently large a 's the inequality

$$
\max _{a \leq t \leq \alpha+\Delta^{2}+\Delta}\|Y(t)\| e^{\left(1+\frac{2}{\Delta}\right)|L| t} \geqq c_{2}
$$

with a positive constant $c_{2}$ -

The proof will be based on the following theorem $\left({ }^{2}\right)$.

If for the complex $w_{1}, w_{2}, \ldots, w_{n}$-numbers we have

$$
\min _{j} \operatorname{Re} w_{j}=0
$$

then for $a>0, d>0$ we have for arbitrary complex $w_{j}$ 's the inequality

holds.

$$
\begin{gathered}
\max _{a \leq t \leq a+d}\left|\sum_{j=1}^{n} b_{j} e^{* w_{j} t}\right| \geqq \\
\geqq\left(\frac{d}{2 e(a+d)}\right)^{n}, \sum_{j=1}^{n} b_{j} \mid
\end{gathered}
$$

As the simple example $\frac{d y}{d t}+L y=0$ (i.e. $n=1$ ) shows, the theorem is in a certain sense best-possible.

2. Next we turn to the proof of our theorem.

With our $Y(t)$ we consider first the system

$$
\frac{d U}{d t}=\boldsymbol{A} U, \quad U(\omega)=Y(\omega)
$$

(') See my book, Eine neue Methode in der Analysis und deren Anwendungen, Budapest, Akad. Kiado, 1953, p. 42. A completely rewritten and enlarged English edition is in preparation. 
with a fixed $\omega \geqq 0$ (which will be chosen later suitably). We suppose first that the characteristic roots of $A$ are simple. If

$$
U=\left(\begin{array}{c}
u_{1} \\
u_{2} \\
\vdots \\
u_{n}
\end{array}\right)
$$

then as well-known, we have for $v=1,2, \ldots, n$

$$
u_{\nu}(t)=\sum_{j=1}^{n} b_{j \nu} e^{\lambda_{j} t}
$$

with constant $b_{j v}$ 's. Application of the theorem $(1,9)-(1.10)$ with the $\omega$ in $(2.1)$ to

$$
u_{v}(t+\omega) e^{-L t}=\sum_{j=1}^{n}\left(b_{j \nu} e^{\lambda_{j} \omega}\right) e^{\left(\lambda_{j}-L\right) t}
$$

and

$$
a=\Delta^{2}, \quad d=\Delta
$$

gives for $v=1,2, \ldots, n$

$$
\begin{gathered}
\max _{\omega+\Delta^{2} \leqq \leq \leqq \omega+\Delta^{2}+\Delta}\left|u_{\nu}(t)\right| e^{-L t}=\max _{\Delta^{2} \leqq t \leqq \Delta^{2}+\Delta}\left|u_{\nu}(t+\omega)\right| e^{-I(t+\omega)} \geqq \\
\geqq \frac{e^{-L \omega}}{(2 e(\Delta+\cdot 1))^{n}}\left|\sum_{j=1}^{n} b_{j \nu} e^{\lambda j^{\prime} \omega}\right|=\frac{1}{(2 e(\Delta+1))^{n}}\left|u_{\nu}(\omega)\right| e^{-L \omega}= \\
=\frac{1}{(2 e(\Delta+1))^{n}} \mid Y_{\nu}(\omega) e^{-L \omega}
\end{gathered}
$$

i.e. for $v=1,2, \ldots, n(L$ is negative !)

$$
\max _{\omega+\Delta^{2} \leqq t \leqq \omega+\Delta^{2}+\Delta}\left|u_{v}(t)\right| \geqq \frac{e^{L\left(\Delta^{2}+\Delta\right)}}{(2 e(\Delta+1))^{n}} \mid Y_{y}(\omega) .
$$

This was deduced under the supposition all the $\lambda_{j}$ 's were simple but a trivial limiting process in (2.2) gives its validity without any restrictions upon the $\lambda_{j}$ 's. Since

$$
\|Y(\omega)\|^{2}=\sum_{\nu=1}^{n}\left|Y_{\nu}(\omega)\right|^{2},
$$

there is an index $v_{0}$ with

$$
\left|Y_{y_{0}}(\omega)\right| \geqq \frac{1}{\sqrt{n}}\|Y(\omega)\|=
$$


and thus from (2.2) we get

$$
\begin{gathered}
\max _{\omega+\Delta^{2} \cong \leq \leqq \omega+\Delta^{2}+\Delta}\|\cdot U(t)\| \geqq \max _{\omega+\Delta^{2} \leqq t \leq \omega+\Delta^{2}+\Delta}\left|u_{v_{0}}(t)\right| \geqq \\
\geqq \frac{e^{L\left(\Delta^{2}+\Delta\right)}}{(2 e(\Delta+1))^{n}}\left|Y_{v_{0}}(\omega)\right| \geqq \frac{e^{L\left(\Delta^{2}+\Delta\right)}}{\sqrt{n}(2 e(\Delta+1))^{n}}\|Y(\omega)\| .
\end{gathered}
$$

3 We shall need two well-known lemmas. If $E$ stands for the $n \times n$ unit-matrix and $E^{(k)}$ the $n \times 1$ column-vector whose $k^{\text {th }}$ component is 1 , the others 0 , further

$$
\nabla(t)=\left(\begin{array}{ccc}
v_{11}(t) & \ldots & v_{1 n}(t) \\
\vdots & & \vdots \\
v_{n 1}(t) & \ldots & v_{n n}(t)
\end{array}\right)
$$

where for $k \equiv 1,2, \ldots, n$ the column-vector $V^{(k)}(t)$ (with the elements of the $k^{\text {th }}$ column of (3.1) as components) satisfies the equation

$$
\frac{d V^{(k)}(t)}{d t}=A V^{(k(t)}
$$

with

$$
V^{(k)}(\omega)=E^{(k)},
$$

then we need the Lemma due to Lrouvilile.

LEMMa I. - With the $U(t)$ of $(2.1)$ we have for $t \geqq \omega$ the (vector) equation $\left({ }^{3}\right)$

$$
Y(t)=U(t)+\int_{\omega}^{t} V^{*}(t+\omega-x) W(Y(x), x) d x .
$$

Further we need PerRon's well-known

LEmms II. - If $x_{1} \geqq 0, x_{2} \geqq 0$ and for the vector $R(t)$ the equation

$$
\frac{d R}{d t}=G(t) R
$$

holds, then we have

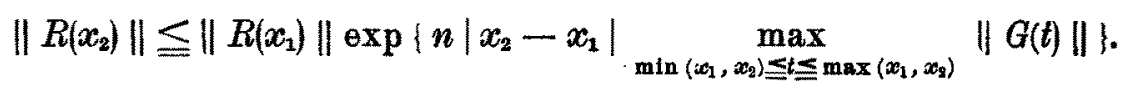

(3) The star denotes the transpose of the matrix in (3.1). 
A simple consequence of Lemma II. which we need is the

Lemma III. - For the $U(t)$ of $(2.1)$ the inequality

$$
\begin{gathered}
\max _{\omega \subseteq t \leq \omega+\Delta^{2}+\Delta}\|U(t)\| \leqq \\
\leqq \exp \left\{n\left(\Delta^{2}+\Delta\right)\|\boldsymbol{A}\|\right\} \max _{\omega+\Delta^{3} \leq t \leq \omega+\Delta^{2}+\Delta}\|U(t)\| .
\end{gathered}
$$

holds.

Let namely $\|U(t)\|$ assume its absolute maximum with respect to $\left[\omega, \omega+\Delta^{2}+\Delta\right]$ at $t=t_{0}$ and with respect to $\left[\omega+\Delta^{2}, \omega+\Delta^{2}+\Delta\right]$ at $t=t_{1}$. We have to apply Lemma II. to $R(t)=U(t)$ with

$$
G(t)=A, \quad x_{1}=t_{1}, \quad x_{2}=t_{0} .
$$

This proves the lemma since

$$
\left|t_{1}-t_{0}\right| \leqq \Delta^{2}+\Delta
$$

4. Now we can turn to the proof of our theorem. Introducing for an $n \times n$ matrix $H=\left(h_{i k}(t)\right)$ a second norm $\|H\|$ by

$$
\|H\|=\left(\sum_{i, k}\left|h_{i k}(t)\right|^{2}\right)^{1 / 2}
$$

(3.4) gives for $\omega \leqq t \leqq \omega+\Delta^{2}+\Delta$ the estimation

$$
\begin{gathered}
\|Y(t)-U(t)\|^{2} \leqq \int_{\omega}^{t}\left\|V^{*}(t+\omega-x)\right\|^{2} \cdot\|W(Y(x), x)\|^{2} d x= \\
=\int_{\omega}^{t}\|V(t+\omega-x)\|^{2} \cdot\|W(Y(x), x)\|^{2} d x .
\end{gathered}
$$

Since applying Lemma II. to the vector $V^{(k)}(t)$ in $(3.2)-(3.3)$ with $x_{1}=\omega$, $x_{2}=y \geqq \omega$ we get

$$
\left\|V^{(k)}(y)\right\|^{2} \leqq e^{2 n\|A,\|(y-\omega)}
$$

i.e. summing over $k=1,2, \ldots, n$

$$
\|V(y)\| \|^{2} \leqq n e^{2 n\|A\|(y-\omega)} .
$$

Owing to $y=t+w-x$ we have now

$$
y-\omega=t-x \leqq t-\omega \leqq \Delta^{2}+\Delta,
$$

and thus we obtained from (4.2) for our $t$ 's

$$
\|Y(t)-U(t)\|^{2} \leqq n e^{2 n\|A\|\left(\Delta^{2}+\Delta\right)} \int_{\omega}^{\omega+\Delta^{2}+\Delta} \| W\left(Y(x), x \|^{2} d x\right.
$$


Owing to $\lim _{t \rightarrow+\infty}\|Y(t)\|=0$ there is a $c_{\mathrm{s}}>0$ such that for $t>c_{\mathrm{s}}$

$$
\|Y(t)\|<c_{1}
$$

with $c_{1}$ from (1.7); choosing

$$
\omega>c_{8}
$$

we get using $(1.8)$ since $(a+b)^{2} \leqq 2\left(a^{2}+b^{2}\right)$

$$
\begin{gathered}
\|Y(t)-U(t)\|^{2} \leqq \frac{1}{9} \frac{e^{-2 n\left(\Delta^{2}+\Delta\right) \| A} \|}{\Delta^{2}+\Delta} \int_{\omega}^{\omega+\Delta^{2}+\Delta}\|Y(x)\|^{2} d x \leqq \\
\leqq \frac{2}{9} \frac{\left.e^{-2 n(\Delta 2}+\Delta\right)\|A\|}{\Delta^{2}+\Delta} \int_{\omega}^{\omega+\Delta^{2}+\Delta}\|U(x)\|^{2} d x+\frac{2}{9\left(\Delta^{2}+\Delta\right)} \int_{\omega}^{\omega+\Delta^{2}+\Delta}\|Y(x)-U(x)\|^{2} d x .
\end{gathered}
$$

This holds for $\omega \leqq t \leqq \omega+\Delta^{2}+\Delta$; if

$$
\max _{\omega \subseteq t \leq \omega+\Delta^{2}+\Delta}\|Y(x)-U(x)\|=\left\|Y\left(t_{2}\right) \leq U\left(t_{2}\right)\right\|,
$$

then applying (4.5) with $t=t_{2}$ results

$$
\begin{aligned}
\left\|Y\left(t_{2}\right)-U\left(t_{2}\right)\right\|^{2} & \leqq \frac{2}{9} e^{-2 n\left(\Delta^{2}+\Delta\right)\|A\|} \max _{\omega \subseteq t \leqq \alpha+\Delta^{2}+\Delta}\|U(t)\|^{2}+ \\
& +\frac{2}{9}\left\|Y\left(t_{2}\right)-U\left(t_{2}\right)\right\|^{2} .
\end{aligned}
$$

Hence

$$
\left\|Y\left(t_{2}\right)-U\left(t_{2}\right)\right\|^{2} \leqq \frac{2}{7} e^{-2 n\left(\Delta^{2}+\Delta\|A\|\right.} \max _{\omega \leq t \leq \omega+\Delta_{2}+\Delta}\|U(t)\|^{2}
$$

and using Lemma III. we obtained

$$
\left\|Y\left(t_{2}\right)-U\left(t_{2}\right)\right\|^{2} \leqq \frac{2}{7} \max _{\omega+\Delta^{2} \leqq t \leq \omega^{2}+\Delta^{2}+\Delta}\|U(t)\|^{2} .
$$

If this last maximum is attained for $t=t_{3}$, then from (4.6) we get

$$
\begin{gathered}
\max _{\substack{\omega+\Delta^{2} \leq t \leq \omega+\Delta^{2}+\Delta \\
\geqq}}\|Y(t)\|^{2} \geqq\left\|Y\left(t_{3}\right)\right\|^{2} \geqq \\
\geqq \frac{1}{2}\left\|U\left(t_{3}\right)\right\|^{2}-\left\|Y\left(t_{3}\right)-U\left(t_{3}\right)\right\|^{2} \geqq \frac{1}{2}\left\|U\left(t_{3}\right)\right\|^{2}-\left\|Y\left(t_{2}\right)-U\left(t_{2}\right)\right\|^{2} \geqq \\
\geqq \frac{1}{2}\left\|U\left(t_{3}\right)\right\|^{2}-\frac{2}{7}\left\|U\left(t_{3}\right)\right\|^{2}=\frac{3}{14} \max _{\omega+\Delta^{2} \leqq t \leq \omega+\Delta^{2}+\Delta}\|U(t)\|^{2}> \\
>\frac{1}{5 n} \frac{e^{2 L\left(\Delta^{2}+\Delta\right)}}{(2 e(\Delta+1))^{2 n}}\|Y(\omega)\|^{2}
\end{gathered}
$$


owing to (2.3). Using (1.6) this gives finally from (4.7) and (1.6)

$$
\max _{\omega+\Delta^{2} \leq t \leq \omega+\Delta^{2}+\Delta}\|Y(t)\|>e^{L\left(\Delta^{2}+2 \Delta\right)}\|Y(\omega)\| \text {. }
$$

5. From (4.8) the theorem follows at once. Namely if $Y(t)$ does not vanish identically for $t>c_{3}$, then there is an $\omega_{0}>c_{s}$ such that

$$
\text { \| } Y\left(\omega_{0}\right) \|>0 \text {. }
$$

We apply first (4.8) with $\omega=\omega_{0}$. This gives the existence of a $t=\omega_{1}$ with

and

$$
\omega_{0}+\Delta^{2} \leqq \omega_{1} \leqq \omega_{0}+\Delta^{2}+\Delta
$$

$$
\left\|Y\left(\omega_{1}\right)\right\| \geqq e^{L\left(\Delta^{2}+2 \Delta\right)}\left\|Y\left(\omega_{0}\right)\right\| \text {. }
$$

Next we apply (4.8) with $\omega=\omega_{1}$. This gives the existence of a $t=\omega_{2}$ with

and

$$
\omega_{1}+\Delta^{2} \leqq \omega_{2} \leqq \omega_{1}+\Delta^{2}+\Delta
$$

$$
\begin{gathered}
\left\|Y\left(\omega_{2}\right)\right\|>e^{L\left(\Delta^{2}+2 \Delta\right)}\left\|Y\left(\omega_{1}\right)\right\|> \\
>e^{2 L\left(\Delta^{2}+2 \Delta\right)}\left\|Y\left(\omega_{0}\right)\right\| .
\end{gathered}
$$

Continuing this process we get a sequence $\left(0 \leqq \omega_{0}<\omega_{1}<\omega_{2}<\ldots\right.$ with

$$
\omega_{\nu}+\Delta^{2} \leqq \omega_{\nu+1} \leqq \omega_{\nu}+\Delta^{2}+\Delta
$$

and

$$
\left\|Y\left(\omega_{\nu}\right)\right\|>e^{\nu L\left(\Delta^{2}+2 \Delta\right)}\left\|Y\left(\omega_{0}\right)\right\|
$$

for $v=0,1,2, \ldots$. Since from $(5.1)$ we have

$$
\begin{gathered}
\omega_{\nu} \geqq \omega_{0}+v \Delta^{2} \\
\left(\Delta^{2}+2 \Delta\right)_{\nu} \leqq\left(1+\frac{2}{\Delta}\right)\left(\omega_{\nu}-\omega_{0}\right)
\end{gathered}
$$

and, since $L$ is negative,

$$
{ }_{v} L\left(\Delta^{2}+2 \Delta\right) \geqq\left(1+\frac{2}{\Delta}\right) L\left(\omega_{y}-\omega_{0}\right) .
$$

Hence for $\alpha>\omega_{0}$ every interval

$$
\alpha \leqq t \leqq \alpha+\Delta^{2}+\Delta
$$

contains at least one of the $\omega_{y}$ 's and

$$
\left\|Y\left(\omega_{v}\right)\right\| \geqq\left(\left\|Y\left(\omega_{0}\right)\right\| e^{-\left(1+\frac{2}{\Delta}\right) L \omega_{0}}\right) e^{\left(1+\frac{2}{\Delta}\right) L \omega_{\nu}}
$$

i.e.

$$
c_{2}=\left\|Y\left(\omega_{0}\right)\right\| e^{\left(1+\frac{2}{4}\right)|L| \omega_{0}}
$$

can be chosen in the theorem. 